\title{
Compact mode-locked Er-doped fiber laser for broadband cavity-enhanced spectroscopy
}

\author{
Aleksander Głuszek ${ }^{1}$ (D) - Francisco Senna Vieira ${ }^{2}$. Arkadiusz Hudzikowski · Adam Wąż ${ }^{1}$. Jarosław Sotor ${ }^{1}$ (i) . \\ Aleksandra Foltynowicz ${ }^{2} \cdot$ Grzegorz Sobon $^{1}$ (1)
}

Received: 28 April 2020 / Accepted: 9 July 2020 / Published online: 25 July 2020

(c) The Author(s) 2020

\begin{abstract}
We report the design and characteristics of a simple and compact mode-locked Er-doped fiber laser and its application to broadband cavity-enhanced spectroscopy. The graphene mode-locked polarization maintaining oscillator consumes less than $5 \mathrm{~W}$ of power. It is thermally stabilized, enclosed in a 3D printed box, and equipped with three actuators that control the repetition rate: fast and slow fiber stretchers, and metal-coated fiber section. This allows wide tuning of the repetition rate and its stabilization to an external reference source. The applicability of the laser to molecular spectroscopy is demonstrated by detecting $\mathrm{CO}_{2}$ in air using continuous-filtering Vernier spectroscopy with absorption sensitivity of $5.5 \times 10^{-8} \mathrm{~cm}^{-1}$ in $50 \mathrm{~ms}$.
\end{abstract}

\section{Introduction}

Optical frequency combs emerged as powerful tools for molecular spectroscopy due to their high spectral brightness, broad spectral coverage, and compatibility with enhancement cavities [1]. In trace gas sensing, frequency combs allow detection of multiple species with high sensitivity and selectivity in short acquisition times [2-4]. Therefore there is an ongoing effort to harness the potential of comb spectroscopy for field-deployable gas detection platforms. Operation outside the laboratory requires compact, low-cost and low power consumption comb sources, which are enabled by the use of fiber-based technology [5, 6]. Recently a fully self-referenced frequency comb based on an Er-doped fiber laser was reported, with electrical power consumption of only $5 \mathrm{~W}$ [7]. It has been also proven that fiber-based frequency combs are ready for space travel and resistant to microgravity conditions [8]. Moreover, near-infrared fiber

Aleksander Głuszek

aleksandra.foltynowicz@umu.se

Grzegorz Soboń

grzegorz.sobon@pwr.edu.pl

1 Laser and Fiber Electronics Group, Faculty of Electronics, Wrocław University of Science and Technology, Wybrzeze Wyspianskiego 27, 50-370 Wrocław, Poland

2 Department of Physics, Umeå University, 90187 Umeå, Sweden lasers can be used as pumps for compact mid-infrared combs based on nonlinear conversion [3, 9].

The technique that has so far been most successfully used for broadband open path sensing is near and mid-infrared dual-comb spectroscopy (DCS) using both laboratory-based and field deployed platforms based on Er-doped fiber combs and difference frequency generation sources [10-13]. Open path sensing in the mid-infrared was also demonstrated using femtosecond optical parametric oscillator in combination with a VIPA spectrometer [14] or a Fourier transform spectrometer $[15,16]$. However, a field-deployed platform for broadband in situ sensing has only been demonstrated in the UV range using a system based on a frequency-doubled femtosecond Ti:Sapphire laser, an enhancement cavity, and low-resolution compact spectrograph [17, 18]. Similar demonstrations in the near- and mid-infrared range are missing. In situ detection requires path enhancement in the form of a multipass cell or enhancement cavity. Cavity-enhanced Fourier transform spectroscopy, either using DCS [19] or mechanical spectrometers [20], requires tight comb-cavity locking [21] and, in the case of DCS, either complex stabilization schemes [22] or adaptive sampling [23]. An alternative cavity-enhanced comb based technique uses Vernier filtering of the comb by the cavity [24-27]. In particular, the so-called continuous-filtering Vernier spectroscopy (CF-VS) $[27,28]$, allows recording cavity enhanced molecular spectra over the entire spectral bandwidth of the femtosecond laser in tens of ms. In CF-VS, an offset is introduced between the repetition rate of the laser $\left(f_{\text {rep }}\right)$ and the external cavity 
free spectral range (FSR) to generate a series of transmission peaks within the laser spectrum - the so-called Vernier orders (VO). The width of each VO, which determines the spectral resolution, depends on the $f_{\text {rep }} /$ FSR mismatch. The VOs are swept across the laser spectrum by scanning either the laser $f_{\text {rep }}$ or the cavity FSR, and a selected VO is recorded in cavity transmission by a single detector. Scanning the $f_{\text {rep }}$ has the advantage that it allows using monolithic fixedlength cavities, with reduced risk of misalignment during operation. Slow and wide range $f_{\text {rep }}$ control is needed to introduce the initial $f_{\text {rep }} /$ FSR offset (usually of the order of a few $\mathrm{kHz}$ ), and faster sweep of $f_{\text {rep }}$ (at a rate of few tens of $\mathrm{Hz}$ with an amplitude of a few hundred $\mathrm{Hz}$ ) is needed for spectral acquisition. The carrier envelope offset frequency of the laser does not need to be controlled. Therefore, CF-VS has the potential to be used in combination with compact mode-locked fiber lasers.

Here we report a simple and compact $125 \mathrm{MHz}$ modelocked Er-doped fiber laser with wide $f_{\text {rep }}$ tuning capabilities and its application to broadband cavity-enhanced continuous-filtering Vernier spectroscopy. The laser is equipped with three different actuators for tuning of the $f_{\text {rep }}$, based on PZT fiber stretchers and a metal-coated fiber section acting as a resistive heater. The PZTs enable $f_{\text {rep }}$ tuning by $2.6 \mathrm{kHz}$ in total and locking it to an external reference with relative rms of the noise of $0.42 \mathrm{mHz}$ over $>20 \mathrm{~h}$ of measurement. The applicability of the laser to broadband and sensitive spectroscopy was verified by detection of $\mathrm{CO}_{2}$ in laboratory air using a CF-VS spectrometer, with absorption sensitivity similar to that obtained with a commercial Er-doped fiber frequency comb.

\section{Laser setup and characteristics}

The setup of the compact Er-doped fiber laser is depicted in Fig. 1a. The laser is built entirely of polarization maintaining (PM) fibers and components. The resonator consists of a filter-type wavelength division multiplexer (PM WDM), a hybrid component comprising an output coupler and isolator (OC/Isolator), a graphene-based saturable absorber, two PZTs and a piece of metal-coated optical fiber. The oscillator is pumped by a $980 \mathrm{~nm}$ single-mode laser diode. For efficient thermal stabilization and tuning, the laser is placed on a metal core printed circuit board (MCPCB) heating plate with uniformly spread heating traces, ensuring highly homogenous heat distribution. The whole system (the oscillator with thermal controller) consumes less than $5 \mathrm{~W}$ of power during operation. The resonator together with the MCPCB plate are placed in a 3D-printed enclosure (see Fig. 1b). Two piezo stretchers are used for $f_{\text {rep }}$ tuning: a multilayer piezo stack $(5 \times 5 \times 50 \mathrm{~mm})$ with $2.7 \mu \mathrm{F}$ capacity and $40 \mu \mathrm{m}$ maximum nominal displacement (labeled as "Slow PZT" in Fig. 1), and
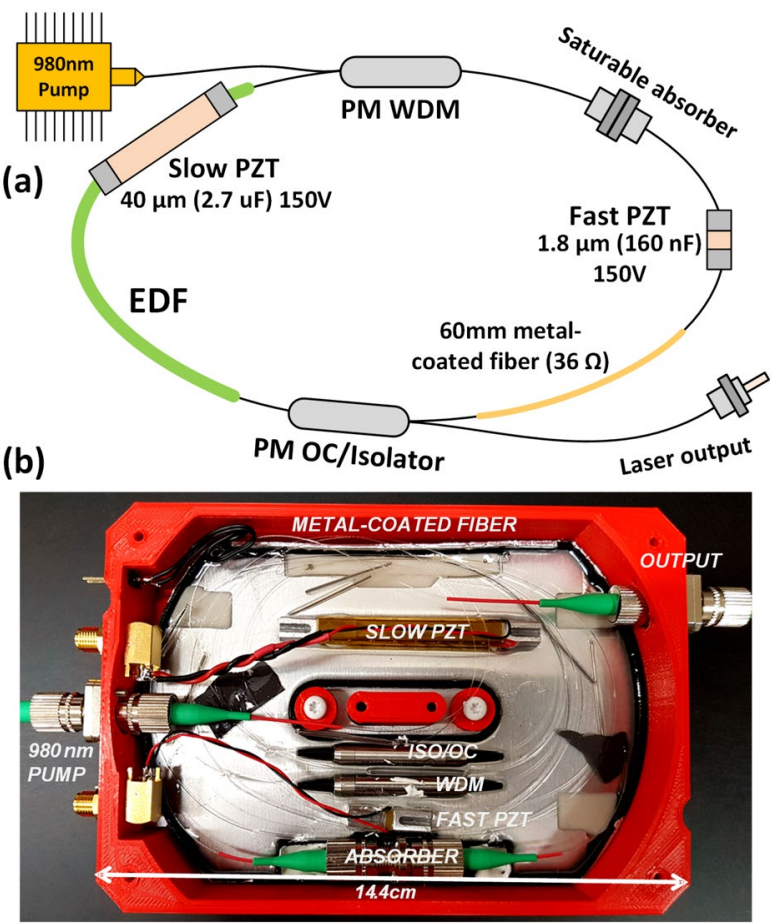

Fig. 1 a Experimental setup of the compact laser oscillator. $P M$ $W D M$ polarization maintaining wavelength division multiplexer, $E D F$ erbium-doped fiber, $P M O C / I S O$ output coupler with isolator, $P Z T$ piezoelectric transducer. b Photograph of the laser in the 3D-printed enclosure

a single piezo chip with $160 \mathrm{nF}$ capacity and $1.8 \mu \mathrm{m}$ nominal displacement (labeled as "Fast PZT" in Fig. 1). Additional tuning is provided by resistive heating of a $60 \mathrm{~mm}$-long segment of fiber coated with a thin layer of metal alloy (Ti/Ni/ Au with $36 \Omega$ resistance and maximum applicable voltage of $2.5 \mathrm{~V}$ ).

The characteristics of the laser are summarized in Fig. 2. Due to the net anomalous dispersion of the resonator, the laser generates a soliton-shaped spectrum with $8.08 \mathrm{~nm}$ full width at half maximum (FWHM) bandwidth centered at $1564 \mathrm{~nm}$ (Fig. 2a). The output power is $4.5 \mathrm{~mW}$ at 93 $\mathrm{mW}$ of pump power. The pulse duration measured with an intensity autocorrelator is $335 \mathrm{fs}$ assuming a $\operatorname{sech}^{2}$ pulse shape (Fig. 2b). Figure 2c shows the radio-frequency (RF) spectrum in the full available span of the spectrum analyzer (Keysight N9010A, 3.6 GHz), indicating stable mode-locking without any parasitic modulations. The pulse repetition rate without any voltage applied to the PZT actuators and the temperature set to $30{ }^{\circ} \mathrm{C}$ is equal to $125.028 \mathrm{MHz}$. The first harmonic of the RF spectrum is shown in Fig. 2d, with signal to noise ratio of more than $70 \mathrm{~dB}$.

Figure 3 shows the static tuning performance of the laser repetition rate using the three available actuators and temperature control. The RF spectra show the first harmonic $(\sim 125.028 \mathrm{MHz})$ recorded for two extreme setpoints of each 

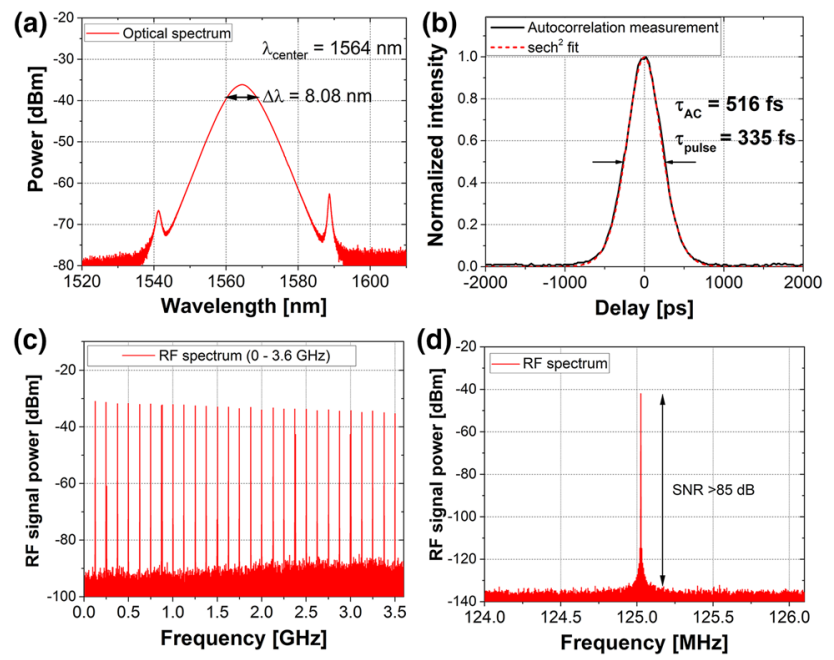

Fig. 2 Characteristics of the oscillator: a optical spectrum measured with $0.01 \mathrm{~nm}$ resolution, $\mathbf{b}$ pulse autocorrelation indicating a duration of $335 \mathrm{fs}$, c radio-frequency spectrum measured with $270 \mathrm{kHz}$ resolution bandwidth and $3.6 \mathrm{GHz}$ frequency span, $\mathbf{d}$ narrow-span RF spectrum showing the first harmonic, measured with $10 \mathrm{~Hz}$ resolution bandwidth
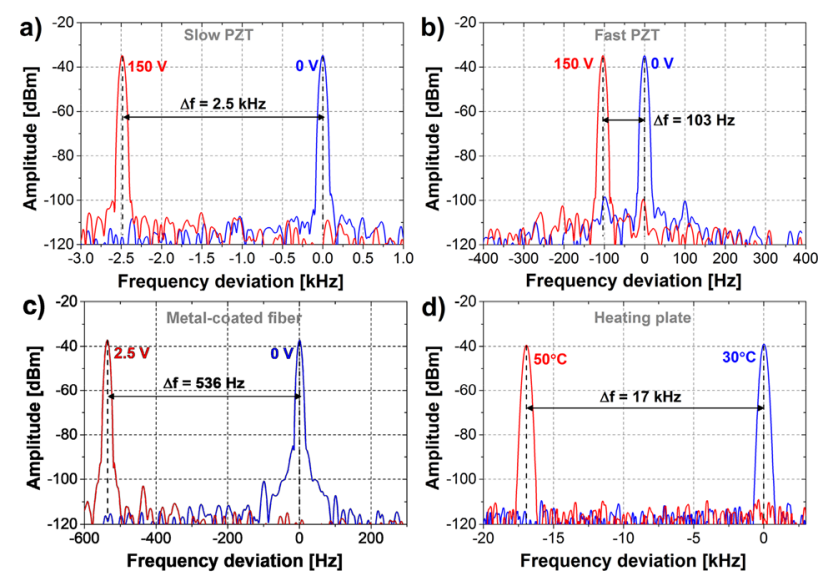

Fig. 3 Static tuning characteristics of the laser repetition rate using various actuators: a slow PZT, b fast PZT, c metal-coated fiber, $\mathbf{d}$ MCPCB heating plate. The zero frequency corresponds to the nominal repetition frequency with no voltage applied to any actuator and the temperature set to $30^{\circ} \mathrm{C}(125.028 \mathrm{MHz})$

actuator. The slow PZT provides elongation of the fiber by $\sim 37 \mu \mathrm{m}$ under maximum driving voltage $(150 \mathrm{~V})$ which corresponds to a repetition rate change of $2.5 \mathrm{kHz}$ (Fig. 3a) and $3.8 \mathrm{GHz}$ comb mode tuning in the optical domain. The fast PZT elongates the fiber by $\sim 1.5 \mu \mathrm{m}$, resulting in $f_{\text {rep }}$ change by $103 \mathrm{~Hz}$ (Fig. 3b) and comb mode shift by $158 \mathrm{MHz}$ in the optical domain. The difference between the nominal PZT displacement and the obtained optical path elongation results from the gluing process, where bond elasticity and piezo pre-tension play the main role. A relatively large tuning range of $536 \mathrm{~Hz}$ (corresponding to $8 \mu \mathrm{m}$ of static stretch) is provided by the metal-coated resistive heater (Fig. 3c) by applying only $2.5 \mathrm{~V}$. The $f_{\text {rep }}$ can be widely tuned by heating the MCPCB plate: by changing the temperature of the laser from 30 to $50{ }^{\circ} \mathrm{C}$ the repetition rate decreases by $17 \mathrm{kHz}$, corresponding to a thermal tuning coefficient of $-0.85 \mathrm{kHz} /{ }^{\circ} \mathrm{C}$.

The frequency response of the actuators was experimentally verified using a fiber-based laser vibrometer, as schematically depicted in Fig. 4a. The vibrometer operates at the wavelength of $1550 \mathrm{~nm}$ [29] and is capable of measuring displacements with resolution down to $1 \mathrm{~nm}$ in the $0-1 \mathrm{MHz}$ frequency range. Figure $4 \mathrm{~b}$ shows the obtained mechanical stretch of the investigated actuators (both PZTs and the metal-coated fiber) as a function of the modulation frequency, expressed in meters per $1 \mathrm{~V}$ of applied voltage. During the measurement, the PZTs were driven with 3.56 $\mathrm{V}_{\mathrm{rms}}$ sine wave from a signal generator while the metalcoated fiber was driven with $1.4 \mathrm{~V}_{\mathrm{rms}}$. The measurement revealed a resonance for both PZTs at $8 \mathrm{kHz}$ and a flat response between 0 and $5 \mathrm{kHz}$. The response of the metalcoated fiber heater decreases with increasing frequency, however, the maximum possible thermally induced stretching of the $60 \mathrm{~mm}$ coated segment of fiber is at the level of $8 \mu \mathrm{m}$ for static conditions.

The stability of the oscillator was investigated by measuring the repetition frequency over a period of $22 \mathrm{~h}$ in two cases: without active $f_{\text {rep }}$ stabilization (with only thermal stabilization of the MCPCB at $32{ }^{\circ} \mathrm{C}$ ) and with $f_{\text {rep }}$ locked to an external reference. The passive long term stability
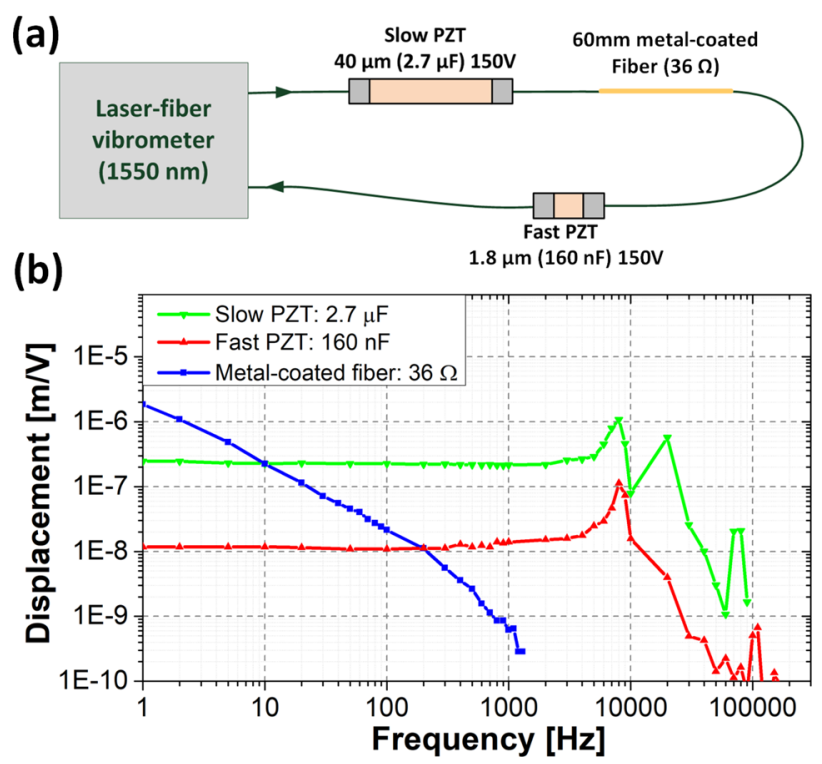

Fig. 4 Actuator bandwidth measurement using laser-fiber vibrometer: a experimental setup, $\mathbf{b}$ response of the investigated actuators per one volt as a function of the modulation frequency 

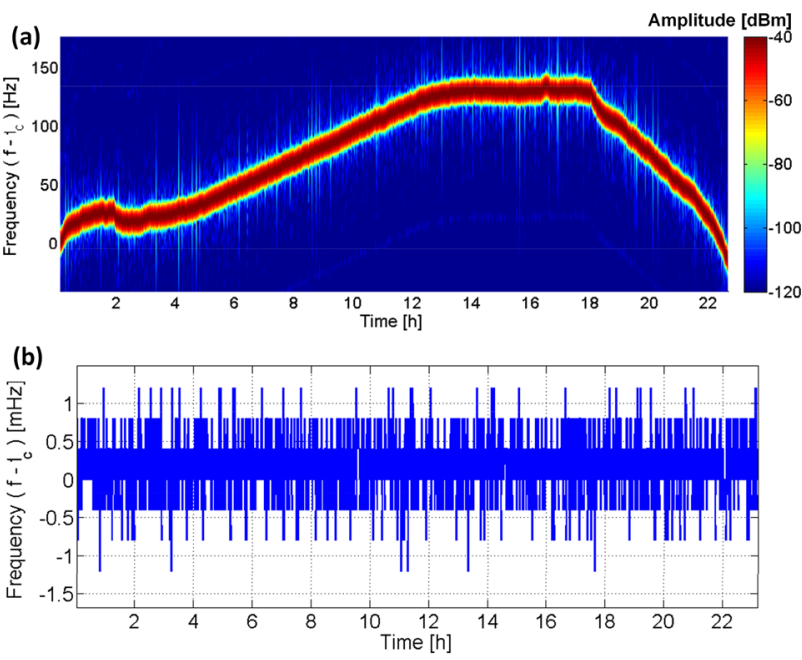

Fig. 5 Repetition frequency measured over $22 \mathrm{~h}$ with: a a freerunning laser (with only thermal stabilization of the cavity at $32{ }^{\circ} \mathrm{C}$ ), $\mathbf{b}$ laser stabilized to an RF reference. $f_{\mathrm{c}}$ center frequency of $125.028 \mathrm{MHz}$

measurement is plotted in Fig. 5a. The $f_{\text {rep }}$ was measured by an RF signal analyzer (Keysight EXA N9010A) referenced to its internal clock source $\left( \pm 2 \mathrm{ppm}\right.$ in the $20-30{ }^{\circ} \mathrm{C}$ range). Data was acquired every $30 \mathrm{~s}$ with $10 \mathrm{~Hz}$ resolution bandwidth (RBW). It can be observed that the simple temperature stabilization based on the MCPCB board and a 3D-printed enclosure provides a very good stability of the $f_{\text {rep }}$ (amplitude of the drift does not exceed $150 \mathrm{~Hz}$ ). A stability improvement can be observed between hours 12-18 of the measurement, which corresponds to night hours (2 am-8 am) and a steady temperature in the laboratory. The usual fluctuation of the temperature in the laboratory is within $1{ }^{\circ} \mathrm{C}$. The frequency variation is caused mainly by the thermal instabilities of the oscillator (the temperature controller stability, temperature gradient in the laser enclosure, etc.) and the inaccuracy of the internal clock source in the RF analyzer. For active stabilization a signal generator (HP $8648 \mathrm{C}$ ) was locked to the RF analyzer using the $10 \mathrm{MHz}$ reference output. A $125.028 \mathrm{MHz}$ signal was generated and compared with the oscillator $f_{\text {rep }}$ by a phase detector (Menlo DXD200). The error signal was delivered to a servo controller (Vescent Photonics D2-125) that drove the PZT stretchers via a 0-150 V amplifier (Piezomechanik SVR 150/3). For better resolution, the $f_{\text {rep }}$ was measured at the 25 th harmonic (3.126 GHz) and normalized to the first harmonic (i.e. divided by 25). Data points were acquired every $30 \mathrm{~s}$ with $10 \mathrm{~Hz}$ span and $1 \mathrm{~Hz}$ RBW. Figure $5 \mathrm{~b}$ presents the relative stability of the oscillator when the $f_{\text {rep }}$ was locked to the reference clock in the RF analyzer. The rms of the noise is $<0.42 \mathrm{mHz}$ demonstrating the excellent relative stability of the repetition frequency in long term operation. We did not use the metal-coated fiber in the $f_{\text {rep }}$ lock, however, it might be successfully used for simple and low-power thermal stabilization of a mode-locked oscillator, as shown in [7].

\section{Cavity-enhanced Vernier spectroscopy}

The applicability of the compact Er-doped fiber laser to broadband spectroscopy was verified using it as a source for continuous-filtering Vernier spectrometer, depicted in Fig. 6. The light emitted by the oscillator was amplified to $105 \mathrm{~mW}$ in an all-fiber erbium-doped fiber amplifier (EDFA), and simultaneously spectrally broadened via self-phase modulation to cover the absorption band of $\mathrm{CO}_{2}$. The output of the amplifier was sent through a polarization maintaining fiber isolator, coupled to free space and mode-matched to a $L=60 \mathrm{~cm}$ long linear cavity $(F S R=250 \mathrm{MHz}$, finesse $F=1050$ ) by a telescope. The power incident on the cavity was $89 \mathrm{~mW}$ (with power spectral density of $21.5 \mu \mathrm{W} /$ $\mathrm{GHz}$ at $1578 \mathrm{~nm}$ ). The cavity was open to air; to record absorption-free background spectra a $50 \mathrm{~cm}$ tube was placed inside the cavity and filled with $\mathrm{N}_{2}$. A relative mismatch of $6.7 \mathrm{kHz}$ between $f_{\text {rep }}$ and FSR/2, corresponding to a spectral resolution of $4.4 \mathrm{GHz}$, was introduced either by detuning the cavity length by $32 \mu \mathrm{m}$ from the perfect match condition (i.e. $\mathrm{FSR}=2 f_{\text {rep }}$ ) using a translation stage (TS) or by changing the laser heater temperature by $8{ }^{\circ} \mathrm{C}$. The output beam of the cavity was incident on a diffraction grating (DG, 600 grooves/mm) mounted on a galvo-scanner (GS). A half-wave plate $(\lambda / 2)$ placed before the DG was used to

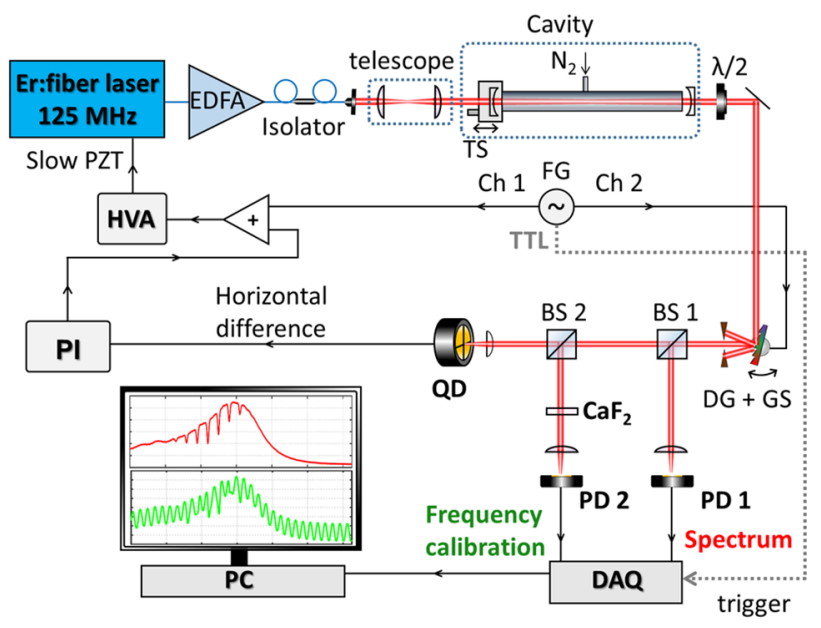

Fig. 6 Experimental setup of the continuous-filtering Vernier spectrometer based on the compact Er-doped fiber oscillator. EDFA Erbium-doped fiber amplifier, $H V A$ high voltage amplifier, $T S$ translation stage, $\lambda / 2$ half-wave plate, $D G$ diffraction grating, $G S$ galvo scanner, $B S 1 \& 2$ beam splitters, $P D 1 \& 2$ InGaAs photodiodes, $Q D$ quadrant detector, $P I$ proportional integral controller, $F G$ function generator, $C h 1 \& 2$ channels of the function generator, $D A Q$ multichannel data acquisition card 


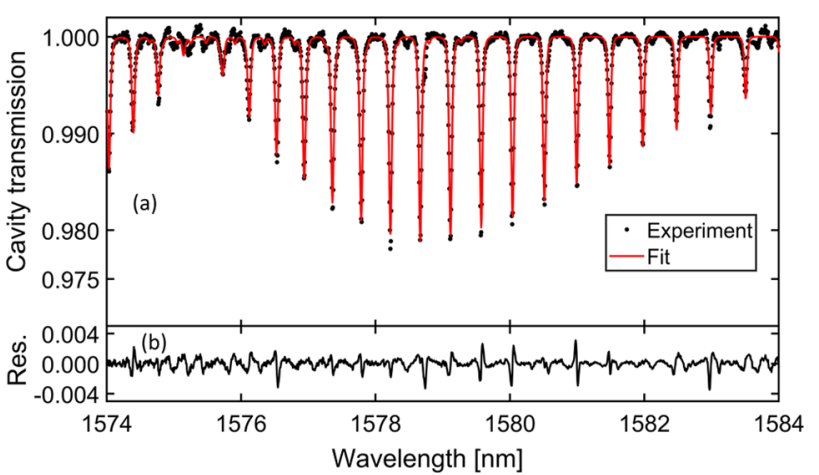

Fig. 7 a Experimental spectrum of the R branch of the $3 v_{1}+v_{3} \mathrm{CO}_{2}$ band in laboratory air (black markers) compared to a fit of a Vernier spectrum model (red line); $\mathbf{b}$ residuum of the fit

adjust the polarization to maximize the diffraction efficiency. The selected VO was swept across the spectrum by applying a $20-\mathrm{Hz}$ sinewave from a function generator (FG) to the laser slow PZT via a high voltage amplifier (HVA). To preserve the propagation direction of the selected VO during the sweep, the GS was simultaneously scanned at the same frequency, with the phase and amplitude of the sinewave adjusted for optimal synchronization. The remaining mismatch was actively corrected by stabilizing the beam direction after the diffraction grating via a feedback loop actuating on the laser $f_{\text {rep }}[27,30,31]$. The diffracted beam was sent through an aperture to block the neighboring VOs, and afterwards split into three arms by two beam splitters (BS $1 \& 2$ ) for spectral acquisition (PD 1, Thorlabs PDA20CS2), frequency calibration $\left(\mathrm{CaF}_{2} \& \mathrm{PD} 2\right.$, Thorlabs PDA20CS2), and stabilization of the spectral scan (QD). The horizontal difference signal of the quadrant detector (QD, Thorlabs PDQ30C) was used as an error signal input to a proportional integral (PI) controller. The correction signal from the PI controller was summed with the sinewave that drove the laser PZT ensuring relative stabilization of the PZT and galvo scans. The peak power incident on the photodetectors PD $1 \& 2$ during the scan was $13 \mu \mathrm{W}$ at $1578 \mathrm{~nm}$, and the detector outputs were recorded by a multichannel data acquisition card (DAQ, NI USB-4432) with $100 \mathrm{kS} / \mathrm{s}$

acquisition rate. The spectrum was resampled at the zero crossings of the $\mathrm{CaF}_{2}$ etalon ( $3-\mathrm{mm}$ long, $\mathrm{FSR}=35 \mathrm{GHz}$ ) fringes, as described in [30], to ensure constant and known sample point spacing. The frequency scale was calibrated by comparing the positions of the absorption lines to a model calculated using a line list from the HITRAN 2016 database [32].

Figure 7a shows a normalized absorption spectrum of $\mathrm{CO}_{2}$ in air (markers) acquired in $1 \mathrm{~s}$ (average of 20 consecutive scans) together with a fit of the CF-VS model $[28,30]$ calculated using a line list from the HITRAN 2016 database, with $\mathrm{CO}_{2}$ concentration as the only fitting parameter. The retrieved $\mathrm{CO}_{2}$ concentration is 694(6) ppm, with the precision given by the standard deviation of the 20 scans. Overall, the data agrees well with the model, and the structure visible in the residual of the fit (Fig. 7b) is mainly caused by inaccuracies in the frequency calibration procedure, as previously observed in [30]. The noise on the baseline, $\sigma$, evaluated as the standard deviation of the ratio of two consecutive background spectra in the spectral range shown in Fig. 7, was $1.1 \times 10^{-3}$. This corresponds to noise equivalent absorption of $5.5 \times 10^{-8} \mathrm{~cm}^{-1}$ in $50 \mathrm{~ms}$, calculated as $\sigma / L_{\text {eff }}$, where $L_{\text {eff }}=\mathrm{FL} / \pi$ is the effective path length in the cavity, equal to $200 \mathrm{~m}$. The corresponding figure of merit is $7.4 \times 10^{-10}$ $\mathrm{cm}^{-1} \mathrm{~Hz}^{-1 / 2}$, calculated as $\sigma T^{1 / 2} /\left(L_{\text {eff }} M^{1 / 2}\right)$, where $\mathrm{T}$ is the acquisition time equal to $50 \mathrm{~ms}$, and $\mathrm{M}$ is the number of spectral elements, given by the ratio of the acquired spectral range $(1.2 \mathrm{THz})$ and the resolution $(4.4 \mathrm{GHz})$. The sensitivity is thus similar to that obtained in a previous implementations of Vernier spectroscopy based on commercial Er-doped fiber frequency combs [25, 31], indicating the potential of our compact source for quantitative and sensitive measurements.

\section{Summary}

We demonstrated a compact and simple mode-locked Erdoped fiber oscillator with active $f_{\text {rep }}$ stabilization and temperature control that consumes less than $5 \mathrm{~W}$ of power. The repetition rate can be widely tuned using three actuators. The implemented PZT stretchers enable efficient locking of the repetition rate to an external reference source, with relative noise RMS value better than $0.42 \mathrm{mHz}$ over $22 \mathrm{~h}$. The applicability of the laser to cavity-enhanced spectroscopy was verified by detection of $\mathrm{CO}_{2}$ in atmospheric air using a continuous-filtering Vernier spectrometer. Ongoing improvements to the Vernier spectrometer that will remove the need for active stabilization of the scan will enable field deployment of the platform for in situ atmospheric measurements.

Acknowledgements Open access funding provided by Umea University. The work was funded by the Foundation for Polish Science (FNP, First TEAM/2017-4/39), the Polish National Agency for Academic Exchange NAWA (PPI/APM/2018/1/00031/U/001), and Knut and Alice Wallenberg Foundation (KAW 2015.0159).

Open Access This article is licensed under a Creative Commons Attribution 4.0 International License, which permits use, sharing, adaptation, distribution and reproduction in any medium or format, as long as you give appropriate credit to the original author(s) and the source, provide a link to the Creative Commons licence, and indicate if changes were made. The images or other third party material in this article are included in the article's Creative Commons licence, unless indicated otherwise in a credit line to the material. If material is not included in the article's Creative Commons licence and your intended use is not permitted by statutory regulation or exceeds the permitted use, you will 
need to obtain permission directly from the copyright holder. To view a copy of this licence, visit http://creativecommons.org/licenses/by/4.0/.

\section{References}

1. N. Picqué, T.W. Hänsch, Nat. Photon. 13, 146-157 (2019)

2. F. Adler, P. Masłowski, A. Foltynowicz, K.C. Cossel, T.C. Briles, I. Hartl, J. Ye, Opt. Express 18, 21861-21872 (2010)

3. G. Ycas, F.R. Giorgetta, E. Baumann, I. Coddington, D. Herman, S.A. Diddams, N.R. Newbury, Nat. Photon. 12, 202-208 (2018)

4. A.V. Muraviev, V.O. Smolski, Z.E. Loparo, K.L. Vodopyanov, Nat. Photon. 12, 209-214 (2018)

5. L.C. Sinclair, J.-D. Deschênes, L. Sonderhouse, W.C. Swann, I.H. Khader, E. Baumann, N.R. Newbury, I. Coddington, Rev. Sci. Instrum. 86, 081301 (2015)

6. L.C. Sinclair, I. Coddington, W.C. Swann, G.B. Rieker, A. Hati, K. Iwakuni, N.R. Newbury, Opt. Express 22, 6996-7006 (2014)

7. P. Manurkar, E.F. Perez, D.D. Hickstein, D.R. Carlson, J. Chiles, D.A. Westly, E. Baumann, S.A. Diddams, N.R. Newbury, K. Srinivasan, S.B. Papp, I. Coddington, OSA Continuum 1, 274-282 (2018)

8. M. Lezius, T. Wilken, C. Deutsch, M. Giunta, O. Mandel, A. Thaller, V. Schkolnik, M. Schiemangk, A. Dinkelaker, A. Kohfeldt, A. Wicht, M. Krutzik, A. Peters, O. Hellmig, H. Duncker, K. Sengstock, P. Windpassinger, K. Lampmann, T. Hülsing, T.W. Hänsch, R. Holzwarth, Optica 3, 1381-1387 (2016)

9. K. Krzempek, D. Tomaszewska, A. Głuszek, T. Martynkien, P. Mergo, J. Sotor, A. Foltynowicz, G. Soboń, Opt. Express 27, 37435-37445 (2019)

10. G.B. Rieker, F.R. Giorgetta, W.C. Swann, J. Kofler, A.M. Zolot, L.C. Sinclair, E. Baumann, C. Cromer, G. Petron, C. Sweeney, P.P. Tans, I. Coddington, N.R. Newbury, Optica 1, 290-298 (2014)

11. S. Coburn, C.B. Alden, R. Wright, K. Cossel, E. Baumann, G.-W. Truong, F. Giorgetta, C. Sweeney, N.R. Newbury, K. Prasad, I. Coddington, G.B. Rieker, Optica 5, 320-327 (2018)

12. G. Ycas, F.R. Giorgetta, K.C. Cossel, E.M. Waxman, E. Baumann, N.R. Newbury, I. Coddington, Optica 6, 165-168 (2019)

13. G. Ycas, F.R. Giorgetta, J.T. Friedlein, D. Herman, K.C. Cossel, E. Baumann, N.R. Newbury, I. Coddington, Opt. Express 28, 14740-14752 (2020)

14. L. Nugent-Glandorf, F.R. Giorgetta, S.A. Diddams, Appl. Phys. B 119, 327-338 (2015)
15. Z. Zhang, R.J. Clewes, C.R. Howle, D.T. Reid, Opt. Lett. 39, 6005-6008 (2014)

16. O. Kara, F. Sweeney, M. Rutkauskas, C. Farrell, C.G. Leburn, D.T. Reid, Opt. Express 27, 21358-21366 (2019)

17. R. Grilli, G. Méjean, S. Kassi, I. Ventrillard, C. Abd-Alrahman, D. Romanini, Environ. Sci. Technol. 46, 10704-10710 (2012)

18. R. Grilli, M. Legrand, A. Kukui, G. Méjean, S. Preunkert, D. Romanini, Geophys. Res. Lett. 40, 791-796 (2013)

19. B. Bernhardt, A. Ozawa, P. Jacquet, M. Jacquey, Y. Kobayashi, T. Udem, R. Holzwarth, G. Guelachvili, T.W. Hansch, N. Picque, Nat. Photon. 4, 55-57 (2010)

20. A. Foltynowicz, T. Ban, P. Masłowski, F. Adler, J. Ye, Phys. Rev. Lett. 107, 233002 (2011)

21. A. Foltynowicz, P. Masłowski, A.J. Fleisher, B.J. Bjork, J. Ye, Appl. Phys. B 110, 163-175 (2013)

22. N. Hoghooghi, R.J. Wright, A.S. Makowiecki, W.C. Swann, E.M. Waxman, I. Coddington, G.B. Rieker, Optica 6, 28-33 (2019)

23. W. Zhang, X. Chen, X. Wu, Y. Li, H. Wei, Photon. Res. 7, 883889 (2019)

24. C. Gohle, B. Stein, A. Schliesser, T. Udem, T.W. Hänsch, Phys. Rev. Lett. 99, 263902 (2007)

25. F. Zhu, J. Bounds, A. Bicer, J. Strohaber, A.A. Kolomenskii, C. Gohle, M. Amani, H.A. Schuessler, Opt. Express 22, 2302623033 (2014)

26. M. Siciliani de Cumis, R. Eramo, N. Coluccelli, M. Cassinerio, G. Galzerano, P. Laporta, P. De Natale, P. Cancio Pastor, Phys. Rev. A 91, 012505 (2015)

27. L. Rutkowski, J. Morville, Opt. Lett. 39, 6664-6667 (2014)

28. L. Rutkowski, J. Morville, J. Quant. Spectrosc. Radiat. Transf. 187, 204-214 (2017)

29. A.T. Waz, G. Dudzik, P.R. Kaczmarek, K.M. Abramski, Photonics Lett. Pol. 6, 133-135 (2014)

30. A. Khodabakhsh, L. Rutkowski, J. Morville, A. Foltynowicz, Appl. Phys. B 123, 210 (2017)

31. C. Lu, F.S. Vieira, F.M. Schmidt, A. Foltynowicz, Opt. Express 27, 29521-29533 (2019)

32. I.E. Gordon et al., J. Quant. Spectrosc. Radiat. Transf. 203, 3-69 (2017)

Publisher's Note Springer Nature remains neutral with regard to jurisdictional claims in published maps and institutional affiliations. 